wenig dichter beschuppten Querbinden sehr undeutlich abheben, die Schenkel sind dünner und alle ohne Spur eines Zähnchens.

Was die Färbung angeht, so variirt dieselbe bei ruber und auch bei anderen Coeliodes-Arten, so dafs die schwarze Stirn und die undeutlich herzförmige schwarze Makel dicht hinter der queren Vertiefung auf dem Thorax möglicherweise nur dem Individuum zukommen.

\title{
VIII. Zur Curculionidenfauna Griechenlands und Cretas.
}

\section{Von}

Johannes Faust.

Das nachfolgende Verzeichnifs ist nach dem Material zusammengestellt, welches mir von Herrn v. Oertzen zur Bestimmung übergeben und welches von ihm auf seinen früheren Reisen in Griechenland gesammelt worden ist. In das folgende Verzeichnifs sind nur diejenigen Arten aufgenommen, welche weder in ${ }$ E. v. Oertzen's Verzeichnifs der Coleopteren Griechenlands und Cretas" noch in dem vorstehenden Artikel erwähnt sind; einige wenige bereits in jenem Verzeichnifs erwähnte Arten sind, weil sie kurz besprochen werden sollen, hier wieder aufgenommen und mit * vor dem Namen versehen.

Phyllobius creticus n.sp. Creta. 1. Sitona cambricus Steph. Creta. - pellitus Bohm. Att., Eub. Tychius comptus Tourn. Attika. Mylacus ${ }_{*}^{*}$ indutus Ksw. ${ }^{1}$ ). 2 . - exiguus n. sp. Morea. 6 . Polydrosus armipes var. secretus ${ }^{2}$ ). - Oertzeni n. sp. Morea. 7.

Creta.

Conocetus gracilis Strl. 3. Psalidium ${ }_{*}^{*}$ maxillosum F. Parnals. 4.

- parnassicum n. sp. Parnafs. 5. Sitona puncticollis Küst. Attika, Morea.
- tenuirostris Tourn. Attika.

- rufovittatus Fst: Att., Samos.

- hirtellus Tourn. Att., Aegina.

- sericans n. sp. Naxos. 8.

- haematopus Gyll. Attika.

Sibinia Heydeni Tourn. Attika. - meridionalis Ch. Bris. Attika.

1) Siehe diese Zeitschrift 1888 , p. 333 .

$\left.{ }^{2}\right)$ Siehe den vorhergehenden Artikel No. 2. 
Gymnetron plagiatum Gyll. Att., Ceutorrhynchus T-album Gyllenh. Morea. Morea.

- thapsicola Germ. Parnafs.

- asellum Germ. Morea.

- Pirazzoli Strl. Aegina.

- rugulosus Hrbst. Creta.

- sanguinipes Chvr. Creta.

- alliariae H. Bris. Attika.

Mecinus Schneideri Kirsch. Aegina.

- longiusculus Boh. Corfu.

- nanus Gyll. Parnafs.

- similis Ch. Bris. Attika.

Ceutorrhynchidius hepathicus Gyll. Rhynchites interpunctatus Steph.

Nauplia. Attika.

Ceutorrhynchus marginatus Payk. Sphenophorus abbreviatus F. Att. Nauplia, Creta. Baris nivalis $\mathrm{H}$. Bris. Parnafs.

Besprechung und Beschreibung der Arten.

1. Phyllobius (Parascytopus) creticus n. sp. Oblongoovatus, convexus, niger, undique sat dense viridi-squamosus ac pube brunnea erecta obsitus; antennis apice rostri, apice tibiiarum tarsisque testaceis; oculis convexis; articulis 3 primis funiculi elongatis secundo longiore, clava elongata angusta; rostro brevi, subconico, capite continuato longitudinaliter obsolete impresso, apice deplanato nudo et punclato; fronte puncto parvo impressa; prothorace quadrato; lateribus subrotundato; elytris humeris breviter rotundatis, parallelis (屯) vel postice parum dilatatis (오), apice acuminato rotundatis, vix striatis; femoribus dente minato armatis. - Long. 4.5-5.5, lat. 1.4-2 mill.

Creta.

Durch die viel kürzere, gewölbtere Form und die abstehende längere Behaarung nimmt sich diese Art etwas fremd neben den gestreckten und flach gewölbten apollinis, taygetanus, pinicola und mirandus aus. Bis auf Kopf, Fühler und die kahle punktirte Rüsselspitze hat creticus mehr Aehnlichkeit mit pilipes, der Kopf mit dem von serripes, aber die Augen gewölbter. Die Fühler haben die Länge von denen desfulvago, sind aber dünner, der Schaft ist kürzer und mehr gekrümmt, Geifselglied 2 etwas länger als 1 und bedeutend länger als 3 . Thorax beim $\sigma^{\top}$ fast länger, beim $f$ so lang als breit, nahezu cylindrisch. Decken in den Schultern reichlich um $\frac{1}{3}$ breiter als der Thorax, der Quere nach ziemlich hoch, der Länge nach beim $\sigma^{\top}$ weniger, beim $\{$ mehr gewölbt, hinten steil abfallend, innerhalb der Schultern leicht eingedrückt. 
Mattgrüne, kurz ovale, zuweilen an der Spitze abgestutzte Schuppen liegen unregelmälsig so dicht nebeneinander, dafs die Deckenskulptur nicht deutlich erkennbar ist, jedoch nicht so dicht, dafs sich die Körpergrundfarbe nicht erkennen liefse. Gemischt sind die Schuppen der Oberseite mit langen und bräunlichen abstehenden Haaren, welche überall nach hinten geneigt sind. Schuppen auf den nur mit einer feinen Dornspitze versehenen Schenkeln länger, lanzettlich.

2. Mylacus indutus Ksw. wird für Holcorrhinus metallicus Desbr. gehalten, wie mir Herr v. Oertzen auf meine Anfrage brieflich bestätigt hat. Soviel mir bekannt, ist überhaupt kein H. metallicus von Desbrochers oder einem anderen Autor beschrieben.

Da aber auf Sicilien eine Varietät des H. parvicollis Seidl. vorkommt, so ist das Vorkommen von Holcorrhinus auf den griechischen Inseln keineswegs ausgeschlossen.

3. Conocetus gracilis Strl. Drei mir als P. gracilicornis Ksw. vorgelegte Stücke von Creta gehören nach Stierlin's Bestimmungstabellen entschieden zu gracilis Strl.; dieselben sind bis auf die braune Körpergrundfarbe, den etwas flacheren Decken, die graugelbe hier und da etwas metallisch glänzende Beschuppung, etwas schmäleren Kopf mit längeren Augen dem gracilicornis Ksw. (graecus Strl.) ähnlich. Die von Dr. Stierlin aufgeworfene Frage, ob gracilis als gute Art (oder als Varietät von graecus) aufzufassen ist, mufs entschieden bejaht ebenso aber auch zugegeben werden, dafs die Beschreibung des Polydrosus marcidus Ksw., welcher in Stierlin's Bestimmungstabellen als Polydrosus mit ovalen Augen aufgenommen ist, nicht schlechter auf diese 3 Stücke pafst. Auf meine Veranlassung verglich Herr v. Oertzen von seinen, mir als gracilicornis gesandten Stücken mit dem typischen marcidus in Dr. Kraatz' Sammlung und theilte mir brieflich mit, dafs er keine wesentlichen Unterschiede zwischen beiden Arten hat feststellen können, dafs ferner die 3 Stücke in der Kraatz'schen Sammlung gelblich grün seien, die in v. Oertzen's Sammlung in der Färbung der Schuppen zwischen gelblich, grau-grün und hellgrün schwanken.

4. Psalidium maxillos um ${ }^{1}$ ) F. Die Stücke vom Parnafs unterscheiden sich nicht von meinen Stücken aus Ungarn, S. Rufs-

1) Ps. interstitiale Boh. ㅇv von Persien, Armenien, dem Araxesthal (Reitter und Leder) ist dieser Art sehr ähnlich, unter- 
land, Caucasus, Kl. Asien. Unter mehr als 60 Exemplaren, welche ich von allen diesen Fundarten gesehen, ist mir auffallenderweise kein einziges $\sigma^{\top}$ vorgekommen. $\mathrm{Zu}$ den charakteristischen Kennzeichen der $q$ dieser Art gehören die weit vorragenden, ziemlich spitzigen Mandibeln, der nach vorn stark gerundet-verengte Thorax mit ganz flachen, die Mehrzahl der Punkte ausfüllenden Schuppen, die von der Basis zur Spitze an Gröfse abnehmenden eingedrückten Punkte in den Deckenreihen, die kleinen runden, stark gewölbten, aber nicht ganz dicht aneinandergestellten Schuppen auf den Spatien, welche diesen ein gekörntes Ansehen geben, und der bis zu den Hinterhüften mit Toment bedeckte Marginalsaum.

5. Psalidium parnassicum. Elongato-oblongum ( $\left.\sigma^{\top}\right)$ vel oblongum ( $(+)$, modice convexum, atrum, subnitidum, squamis fere planis sat dense pilisque adpressis parce obsitum; corpore subtus margine apicali elytrorum albido-tomentosis; rostro quadrato profunde punctato, medio sulcato; antennis crassia scapo valde clavato; prothorace lateribus aequaliter rotundato, antrorsum vix (ठَ) vel evidenter ( $($ ) angustato, dorso punctis remotis mediocris lateribus fossulis profundioribus sat dense obsito; elytris ellipticis basi seriatim punctatis apicem versus punctato-striatis, his interstitiis convexis; pedibus crassiusculis, femoribus subclavatis, tibiis anticis maris apicem versus curvatis et denticulatis. $-\tau$ Long. 7.1, lat. 2.5 mill.; o 8.2 , lat. 3.2 mill.

\section{Parnals.}

Von dem ähnlichen creticum unterscheidet sich die neue Art hauptsächlich durch viel dickere Fühler und Beine, viel weniger dicht punktirten Thorax und den Mangel des Tomentes auf den äufseren Deckenspatien.

Augen, Kopf und Rüssel sind denen von creticum fast gleich, nur fehlt der Stirne über den Augen das Grübchen. Geifselglied 1 doppelt, 2 ebensolang, die übrigen viel kürzer als breit. Thorax so lang als breit, fast ganz wie bei maxillosum skulptirt, die eingestochenen Punkte auf dem Rücken etwas zahlreicher, jeder dieser Punkte mit einem feinen anliegenden gelblichen Härchen. Decken $2 \mathrm{mal}(q)$ oder $2 \frac{1}{2} \mathrm{mal}\left(\sigma^{\top}\right)$ so lang als breit, die Schultern

scheidet sich aber von ihr durch kürzere, dickere Mandibeln, kleinere Augen, längeren Fühlerschaft und Geifsel, dünnere Beine, namentlich sehr viel dünnere Schienen, durch meist etwas kürzere Form und feinere Skulptur, Auch von dieser Art kenne ich nur das ․ 
ganz verrundet, die Seiten gleichmälsig flach gerundet, auf dem Rücken ebenso flach längsgewölbt, aber hinten steiler abfallend als bei creticum; die eingedrückten Punkte in den nur hinten und an den Seiten vertieften Reihen so grofs als bei letzterem, etwas grölser als die auf dem Thoraxrücken und mit einem kurzen feinen Härchen, die Spatien auf dem Rücken sehr flach gewölbt und mit rundlichen, nur flach gewölbten, dunklen, an den Seiten schwarzgrauen Schuppen dicht besetzt, mit eingestreuten, bei gewöhnlicher Vergröfserung nur hinten und an den Seiten sichtbaren kleinen Punkten, welchen ein längeres, wenig abstehendes Härchen entspringt; nur der Marginalsaum hinten bis etwa zum zweiten Bauchsegment mit spärlichem Toment, aber unten dicht gewimpert. Die dicken Schenkel mit deutlichen, tief eingestochenen Punkten. Tarsen deutlich breiter aber nicht kürzer als bei creticum, maxillosum und interstitiale.

Es ist parnassicum diejenige Art, welche in v. Oertzen's Verzeichnifs der Käfer Griechenlands und Cretas für sculpturatum Boh. angesehen ist, kann aber schon deshalb nicht mit diesem verwechselt werden, weil die Decken von sculpturatum profundius punctato-striata interstitiis convexis und die tarsi breves (im Vergleich mit maxillosum und interstitiale) sein sollen.

6. Tychius exiguus. 우. Oblongus, depressus, niger, supra pilis subsquamiformibus albescentibus, subtus squamis ovalibus cretaceis obsitus; antennis, rostri apice, pedibusque testaceis; rostro elongato, recto, lineari, subcylindrico; antennis in medio rostri insertis; oculis vix convexis; prothorace latitudine longiore, apice constricto, antice magis angustato, lateribus paulo rotundato, squamis piliformibus subsericeis sat dense tecto; elytris prothorace paulo latioribus, parallelis, postice angustatis, striatis, in striis serie pilosum tenuissimorum, interstitiis angustis biseriatim pilosis; pedibus tenuioribus, femoribus muticis. - Long. 1.3, lat. 0.6 mill.

Morea. Nur 2 ㅇ.

Noch etwas kleiner als pusillus Germ., namentlich schlanker. Rüssel nicht gebogen und nicht zugespitzt, die ganzen Beine gelb, Thorax länger; von pumilus mit rothen Beinen durch geringere Grölse, schlankere Form, tiefere Deckenstreifen, schmale Spatien, viel spärlichere Behaarung auf diesem, längeren Thorax und viel dünnere Beine zu unterscheiden.

T. perpendus Tourn. vom Libanon mufs in Bezug auf Färbung und Form sehr ähnlich sein, soll aber fein gestreifte Decken, 
breite Spatien und einen zur Spitze verengten Rüssel haben. Auch T. ruficornis Tourn. von derselben Grölse muls dem exiguus nahe stehen, soll aber etwas gebogenen Rüssel (ㅇ), auf der Basalhälfte fast parallele Thoraxseiten und schwarze Schenkel haben.

Die wenig gewölbten Augen fast breiter als die Stirne. Rüssel länger als die Vorderschenkel, reichlich so lang als Kopf und Thorax zusammen. Thoraxvorderrand abgeschnürt und etwas breiter als die halbe, schwach zweibuchtige Basis, die Hinterecken etwas eingezogen, stumpfwinklig aber scharf; die gelblichen etwas seidenglänzenden Schuppen sind lang-lanzettförmig, der Hinterrand mit einer Reihe rundlicher, weifser Schuppen besetzt. Decken mit rechtwinkligen, kurz gerundeten Schultern, etwas breiter als der Thorax, die Seiten bis hinter die Mitte parallel, dann gerundet verengt, die Streifen nicht tief aber breit, nicht schmäler als die Spatien, die in 2 Reihen gestellten Punkte auf diesen gegeneinander versetzt, die Schuppenhaare schmal bandförmig, an der Spitze stumpf. Die weifsen Schuppen der Unterseite sind rundlich und stehen nicht ganz dicht nebeneinander. Die Schenkel sind in den Punkten mit kurzen anliegenden Schuppenhärchen besetzt.

Bei dem einen Stück sind die Decken hinten röthlich angelaufen.

7. Tychius Oertzeni. Sub depressus, ovatus, niger, subtus squamis ovatis dense, supra pilis squamiformibus minus dense obsitus; antennis, rostri apice, tibiis tarsisque testaceis; oculis parum convexis; rostro subrecto aequilato, apicem versus attenuato, basi subtilissimo punctato-strigoso, apice seriatim remoteque punctato; prothorace lateribus modice rotundato, antrorsum magis, basi minus contracto, dense profundeque punctato; elytris parallelis apice obtuse rotundatis, prothorace vix vel haud latioribus, sat profunde striatis, in striis uniseriatim pilosis, interstitiis angustis, pilis squamiformibus minus elongatis biseriatim obsitis, sutura albidiora; femoribus muticis, anticis modice clavatis, tibiis anticis maris denticulo obtuso armatis. - Long. 1.8, lat. 0.7 mill.

\section{Morea.}

Die nächst verwandte Art discicollis Fst. ist gröfser, etwas gewölbter, die längeren Fühler gegen die Spitze schwarz, die Augen gewölbter, der Rüssel beim $q$ kürzer und viel weniger verdünnt, die Behaarung länger. In der Gröfse ist die neue Art dem sericatus Tourn. gleich, aber viel weniger gewölbt, durch längeren und dünneren, viel mehr ausgezogenen Rüssel, grob, tief und dicht punktirten Thorax, hinten nicht verengte Decken, tiefere Streifen, 
schmälere Spatien und viel dünnere Schuppenhaare hauptsächlich verschieden.

Ebenso flache Wölbung und ähnliche Form hat auch comptus, bei diesem sind aber die Augen gewölbter, der Thorax fein punktirt, die Decken hinten weniger stumpf gerundet, die Schienen theilweise schwarz und die Behaarung wohl ebensolang als bei der neuen Art, aber viel feiner.

Stirne kaum so breit als der Durchmesser der weniger gewölbten Augen. Der mit der Stirne überall gleich breite und ziemlich gerade Rüssel so lang ( $\left.\sigma^{\star}\right)$ oder viel länger () als der Thorax, besonders beim $q$ zur Spitze stark verdünnt. Die bis auf die gebräunte Keule ganz gelben Fühler im Spitzendrittel ( $\left.\sigma^{\top}\right)$ oder dicht vor der Mitte ( $($ ) eingefügt. Der Thorax und die Decken sind fast genau so wie die von discicollis punktirt und geformt, ersterer an den Seiten etwas weniger gerundet, letztere mit noch schmäleren Spatien $\mathbf{z w i s c h e n ~ d e n ~ S t r e i f e n , ~ a u f ~ d i e s e m ~ s i n d ~ d i e ~}$ Punkte der beiden Reihen gegeneinander versetzt, weshalb die diesen Punkten entspringenden Schuppenhaare stellenweise in eine unregelmälsige Reihe gestellt und die Spatien dünn beschuppt erscheinen; dagegen stehen die Punkte auf der Sutur nebeneinander und diese erscheint daher dichter beschuppt; die feinen Haare in den Streifen sind so lang und so dünn als diejenigen auf den Spatien von sericatus und comptus.

8. Tychius sericans. Oblongus subcylindricus, niger, subtus albido-squamosus, supra pilis squamiformibus albido-sericeis modice tectus; antennarum basi, rostri apice, tibiis tarsisque testaceis; rostro curvato basi crasso apicem versus aequaliter attenuato ( $\left.\sigma^{\top}\right)$ vel ab insertione antennarum usque ad apicem subulato (ㅇ); prothorace lateribus rotundato, basi bisinuato; elytris parallelis, apice obtuse rotundatis, late striatis, in striis pilis uniseriatim, in interstitiis angustis biseriatim obsitis, sutura albidiori; femoribus muticis, tibiis anticis maris intus unidentatis, tarsis longioribus. - Long. 2.1, lat. 1 mill.

Naxos.

In Form, Gröfse, Färbung der Beine und durch den Zahn der männlichen Vorderschienen steht diese Art dem meliloti nahe, kann aber schon des viel dickeren Rüssels und der Bekleidung wegen nicht gut mit ihm verwechselt werden.

Kopf dicker als bei meliloti; die Augen gröfser und etwas gewölbter, ibre Entfernung etwas breiter als bei diesem. Rüssel an der Wurzel höher als das Auge breit und gleich hier gebogen,

Deutsche Entomol. Zeitschr. 1889. Heft I. 
bis zur Fühlereinlenkung gleichmälsig dünner, aber über die ganze Länge gleich breit. Fühlerschaft und die ersten Geifselglieder hell, die übrigen und die Keule dunkel gefärbt. Thorax so lang als breit, die gerundeten Seiten zur Spitze mehr als zur Basis verengt, die Hinterecken zwar etwas eingezogen, aber scharfwinklig, Rücken dicht punktirt. Decken wenig breiter als der Thorax mit kurz gerundeten Schultern, parallelen Seiten und stumpf gerundeter Spitze, der Länge nach mit dem Thorax gleich und wenig, der Breite nach höher als bei meliloti gewölbt, breit gestreift, die Spatien kaum breiter als die Streifen. Beine wie bei meliloti nur die Tarsen länger.

Die weifslichen, namentlich auf dem Thorax seidenglänzenden Schuppenhaare sind länger und dünner als bei meliloti, stehen auf dem Thorax quer und lassen die Mittellinie desselben, in welcher sie gegeneinander stofsen, etwas dichter behaart erscheinen. Die Schuppenhaare auf den Spatien sind wenig dicker und länger als diejenigen in den Streifen, stehen auf jenen und auf der Sutur gegeneinander versetzt; letztere erscheint namentlich auf der hinteren Hälfte etwas dichter behaart. Auf der Schildchenstelle und am Thoraxhinterrande steht eine Reihe kreideweifser Schuppen von ovaler Form, ähnlich denjenigen auf der Unterseite. 


\section{$2 \mathrm{BHL}$ Biodiversity Heritage Library}

Faust, Johannes. 1889. "VIII. Zur Curculionidenfauna Griechenlands und Cretas." Deutsche entomologische Zeitschrift 1889(1), 91-98. https://doi.org/10.1002/mmnd.48018890111.

View This Item Online: https://www.biodiversitylibrary.org/item/103677

DOI: https://doi.org/10.1002/mmnd.48018890111

Permalink: https://www.biodiversitylibrary.org/partpdf/235428

\section{Holding Institution}

Harvard University, Museum of Comparative Zoology, Ernst Mayr Library

\section{Sponsored by}

Harvard University, Museum of Comparative Zoology, Ernst Mayr Library

\section{Copyright \& Reuse}

Copyright Status: Public domain. The BHL considers that this work is no longer under copyright protection.

This document was created from content at the Biodiversity Heritage Library, the world's largest open access digital library for biodiversity literature and archives. Visit BHL at https://www.biodiversitylibrary.org. 